\title{
Metrik Reissner-Nordström dalam Teori Gravitasi Einstein
}

\author{
Canisius Bernard* \\ Program Studi Fisika, Fakultas Teknologi Informasi dan Sains, \\ Universitas Katolik Parahyangan, Jl. Ciumbuleuit no. 94, Cidadap, Bandung 40141, Indonesia
}

Intisari

Dalam makalah ini akan ditinjau kembali bahwa persamaan medan gravitasi Einstein mempunyai solusi yaitu solusi Reissner-Nordström dengan menghitung seluruh komponen dari tensor Einstein dan tensor energimomentum dengan adanya interaksi elektromagnetik. Solusi Reissner-Nordström adalah solusi yang menggambarkan ruangwaktu di luar sebuah bola pejal statik bermassa $M$ dan bermuatan listrik $Q$. Solusi ReissnerNordström juga merupakan solusi lubang hitam statik bermuatan listrik dalam teori Einstein-Maxwell.

\section{ABSTRACT}

In this paper, we review that Reissner-Nordström metric is the solution of Einstein gravitational field equation. Reissner-Nordström solution describes spacetime outside a static spherically symmetric charged mass. To solve the field equation, we calculate all the non-zero components of Einstein tensor and energy-momentum tensor of the electromagnetic field of the charged object. One finds that the Reissner-Nordström metric is also a charged static black hole solution in Einstein-Maxwell theory.

KATA KUNCI: Persamaan medan Einstein, metrik Reissner-Nordström, lubang hitam http://dx.doi.org/10.12962/j24604682.v13i1.2128

\section{PENDAHULUAN}

Lubang hitam merupakan salah satu fenomena gravitasi yang menarik karena dapat diprediksi oleh penelitian fisika teoretik dan matematika. Adapun cakupan fisis lubang hitam adalah teori gravitasi relativitas umum. Tahun 1915, Einstein menyelesaikan teori relativitas umum, yang merupakan sebuah teori tentang gravitasi [1]. Gravitasi tidak lagi dipandang sebagai suatu gaya, tetapi merupakan perwujudan dari kelengkungan ruangwaktu sendiri. Penggambaran yang lebih lengkap dari gravitasi ini memerlukan suatu persamaan medan yang berlaku secara umum. Persamaan medan yang dimaksud adalah persamaan medan Einstein [2]

$$
G_{\mu \nu}=R_{\mu \nu}-\frac{1}{2} g_{\mu \nu} R=8 \pi T_{\mu \nu}
$$

dengan $R_{\mu \nu}$ adalah tensor Ricci, $g_{\mu \nu}$ adalah tensor metrik, $R$ adalah skalar Ricci, $G_{\mu \nu}$ adalah tensor Einstein, dan $T_{\mu \nu}$ adalah tensor energi-momentum. Persamaan ini adalah suatu persamaan yang menyatakan hubungan antara tensor kelengkungan ruangwaktu (tensor Einstein) dan tensor kehadiran massa atau energi dalam ruangwaktu. Tensor Einstein memberi informasi bahwa ruangwaktu lengkung dan tensor energi-momentum memberi informasi kehadiran massa atau energi dalam ruangwaktu. Jadi persamaan medan Einstein memperlihatkan bahwa setiap benda bermassa mengakibatkan ruangwaktu sekitarnya melengkung. Hal tersebut mempunyai

\footnotetext{
*canisius.bernardegmail.com
}

arti fisis bahwa disekitar benda bermassa akan timbul medan gravitasi. Berbagai solusi eksak dari persamaan medan Einstein sudah berhasil diturunkan. Solusi eksak itu merupakan kelengkungan ruangwaktu suatu lubang hitam atau lebih dikenal dengan istilah metrik kelengkungan ruangwaktu.

Mencari solusi dari persamaan medan Einstein pada dasarnya sangatlah sulit. Pada awalnya, Einstein sendiri mengantisipasi bahwa sangat sukar (atau terkadang tidak mungkin) diperoleh suatu solusi eksak terhadap persamaan medannya. Akan tetapi, cukup mengejutkan bahwa pada tahun 1916, kurang dari setahun setelah publikasi teori relativitas umum, Karl Schwarzschild, di medan perang JermanRussia pada Perang Dunia I, berhasil mendapatkan solusi eksak yang pertama dari persamaan medan Einstein. Kelengkungan ruangwaktu Schwarzschild atau yang dalam jargon fisikawan disebut metrik Schwarzschild adalah solusi lubang hitam statik yang tidak berputar dan mempunyai simetri bola [3]. Yang dimaksud dengan statik adalah dalam sistem koordinat yang digunakan, tensor metriknya tidak bergantung waktu.

Pada tahun 1916 dan 1918, Reissner dan Nordström juga menemukan solusi lubang hitam yang tidak berputar tetapi mempunyai muatan listrik dan bersimetri bola $[4,5]$. Tahun 1963, Roy Kerr mendapatkan solusi untuk lubang hitam yang berputar tetapi tidak bermuatan listrik [6]. Tahun 1965, Ezra "Ted" Newman mendapatkan solusi untuk lubang hitam yang berputar dan bermuatan listrik [7, 8]. Jadi metrik Schwarzschild, metrik Reissner-Nordström, metrik Kerr, dan metrik Kerr-Newman merupakan solusi dari persamaan medan Einstein. Fakta dimana setiap lubang hitam dapat dibedakan berdasarkan hanya tiga parameter fisis, yaitu 
massanya, momentum sudutnya, dan muatan listriknya adalah juga sifat alamiah lubang hitam yang menghilangkan informasi yang masuk padanya. Keadaan tersebut dikenal sebagai teorema "no-hair", berlaku untuk semua lubang hitam yang merupakan solusi dari persamaan medan Einstein-Maxwell tentang gravitasi dan elektromagnetik dalam teori relativitas umum. Adapun fisikawan yang menggagas teorema "no-hair" ini adalah John Archibald Wheeler lebih dari empat puluh tahun yang lalu [9].

Dalam makalah ini, kami akan meninjau kembali ruangwaktu Reissner-Nordström, dimana terdapat kehadiran medan lain, yang berarti bahwa tensor energi-momentum tidak nol. Secara khusus, kami akan berkonsentrasi pada salah satu situasi yang menarik secara fisis yang dalam hal ini tensor energimomentum dari materi harus disertakan dalam persamaan medan Einstein. Kami mempertimbangkan geometri ruangwaktu di luar sebuah massa yang diam dan bermuatan listrik yang mempunyai simetri berbentuk bola. Sekali lagi hal yang akan dibahas ini bukanlah ruang vakum, karena adanya medan listrik dalam ruang. Dalam makalah ini, digunakan unit dengan $G=c=\hbar=4 \pi \epsilon_{0}=1$.

\section{GEOMETRI REISSNER-NORDSTRÖM}

Persamaan Einstein-Maxwell dapat diturunkan dari aksi $[10,11]$

$$
S=\int d^{4} x \sqrt{-g}\left(R-F_{\mu \nu} F^{\mu \nu}\right),
$$

dengan $g$ adalah determinan dari tensor metrik $g_{\mu \nu}, R$ dalam aksi Einstein-Maxwell (Pers.(2)) adalah skalar Ricci, yaitu $R=R_{\mu}^{\mu}=g^{\mu \nu} R_{\mu \nu}$ dan $F_{\mu \nu}$ adalah tensor kuat medan dalam ruang lengkung yang dituliskan sebagai [2]

$$
F_{\mu \nu}=\nabla_{\mu} A_{\nu}-\nabla_{\nu} A_{\mu}
$$

Memvariasi aksi (Pers.(2)) terhadap $g^{\mu \nu}$ menghasilkan persamaan medan Einstein-Maxwell

$$
G_{\mu \nu}=8 \pi T_{\mu \nu} .
$$

dimana $G_{\mu \nu}$ adalah tensor Einstein dan $T_{\mu \nu}$ adalah tensor energi-momentum Maxwell yaitu [12]

$$
T_{\mu \nu}=\frac{1}{4 \pi}\left(g^{\gamma \lambda} F_{\mu \gamma} F_{\mu \lambda}-\frac{1}{4} g_{\mu \nu} F_{\alpha \beta} F^{\alpha \beta}\right) .
$$

Salah satu solusi dari persamaan medan (Pers.(4)) adalah metrik Reissner-Nordström yang menggambarkan ruangwaktu di luar sebuah bola pejal bermassa $M$ dan bermuatan $Q$

$$
\begin{aligned}
d s^{2}= & -\left(1-\frac{2 M}{r}+\frac{Q^{2}}{r^{2}}\right) d t^{2}+\left(1-\frac{2 M}{r}+\frac{Q^{2}}{r^{2}}\right)^{-1} d r^{2} \\
& +r^{2}\left(d \theta^{2}+\sin ^{2} \theta d \phi^{2}\right) .
\end{aligned}
$$

Dalam ruangwaktu ini terdapat vektor potensial $\left[A_{\mu}\right]=$ $\left(A_{0}, A_{1}, A_{2}, A_{3}\right)$ dengan

$$
A_{0}=-\frac{Q}{r},
$$

dan $A_{i}=0$ untuk $i=1,2,3$. Dalam menulis metrik (Pers.(6)), digunakan koordinat $x^{0}=t, x^{1}=r, x^{2}=\theta$, dan $x^{3}=\phi$.

Untuk menghitung tensor Einstein, dibutuhkan seluruh komponen simbol Christoffel yang tak nol dari metrik (Pers.(6)) yaitu

$$
\begin{aligned}
& \Gamma_{01}^{0}=\frac{M r-Q^{2}}{r \Delta}, \Gamma_{00}^{1}=\frac{\left(M r-Q^{2}\right) \Delta}{r^{5}}, \\
& \Gamma_{11}^{1}=-\frac{M r-Q^{2}}{r \Delta}, \Gamma_{22}^{1}=-\frac{\Delta}{r}, \\
& \Gamma_{33}^{1}=-\frac{\Delta \sin ^{2} \theta}{r}, \Gamma_{12}^{2}=r^{-1}, \\
& \Gamma_{33}^{2}=-\sin \theta \cos \theta, \Gamma_{13}^{3}=r^{-1}, \Gamma_{23}^{3}=\cot \theta,
\end{aligned}
$$

dengan

$$
\Delta=r^{2}-2 M r+Q^{2} .
$$

Metrik (Pers.(6)) mempunyai solusi lubang hitam, dimana posisi horison peristiwa adalah solusi radius dari persamaan

$$
g_{r r}^{-1}=\left(1-\frac{2 M}{r}+\frac{Q^{2}}{r^{2}}\right)=0,
$$

yang menghasilkan

$$
r_{ \pm}=M \pm \sqrt{M^{2}-Q^{2}},
$$

dengan $r_{+}$adalah horison peristiwa bagian luar, dan $r_{-}$adalah horison peristiwa bagian dalam. Horison peristiwa adalah sebuah permukaan yang menutup secara total titik singularitas.

\section{A. Tensor Kuat Medan}

Perlu diketahui sebelumnya bahwa tensor kuat medan $F_{\mu \nu}$ pada ruang lengkung sama dengan ruang datar. Dengan mengingat turunan kovarian $\nabla_{a} v_{b}=\partial_{a} v_{b}-\Gamma_{b a}^{c} v_{c}$ dan simbol Christoffel $\Gamma_{b a}^{c}$ yang simetrik, maka Pers.(3) menjadi

$$
F_{\mu \nu}=\partial_{\mu} A_{\nu}-\partial_{\nu} A_{\mu} .
$$

Menggunakan Pers.(12) dapat dihitung komponen tensor kuat medan untuk ruang lengkung yang tak nol yaitu

$$
F_{01}=-F_{10}=\partial_{0} A_{1}-\partial_{1} A_{0}=-\frac{Q}{r^{2}} .
$$

Komponen-komponen matriks $\left[F_{\alpha \beta}\right]$ untuk medan Maxwell yang mengisi ruangwaktu (Pers.(6)) adalah

$$
F_{\alpha \beta}=\left(\begin{array}{cccc}
0 & -\frac{Q}{r^{2}} & 0 & 0 \\
\frac{Q}{r^{2}} & 0 & 0 & 0 \\
0 & 0 & 0 & 0 \\
0 & 0 & 0 & 0
\end{array}\right) .
$$

Menghitung produk skalar $F_{\alpha \beta} F^{\alpha \beta}$ juga diperlukan, yaitu

$$
F_{\alpha \beta} F^{\alpha \beta}=g^{\alpha c} g^{\beta d} F_{\alpha \beta} F_{c d}=-\frac{2 Q^{2}}{r^{4}} .
$$

Informasi yang dibutuhkan selanjutnya adalah seluruh komponen tensor Einstein $G_{\mu \nu}$ yang dapat dicari dengan menghitung tensor Ricci $R_{\mu \nu}$ dan skalar Ricci $R$. 


\section{B. Tensor Ricci dan Skalar Ricci}

Dengan tensor Riemann

$$
R_{\alpha \beta \gamma}^{\mu}=\partial_{\beta} \Gamma_{\alpha \gamma}^{\mu}-\partial_{\gamma} \Gamma_{\alpha \beta}^{\mu}+\Gamma_{\alpha \beta}^{\mu} \Gamma_{\gamma \alpha}^{\sigma}-\Gamma_{\sigma \gamma}^{\mu} \Gamma_{\beta \alpha}^{\sigma},
$$

dan tensor Ricci

$$
R_{\mu \nu}=-R_{\mu \alpha \nu}^{\alpha}
$$

dapat dihitung seluruh komponen tensor Ricci untuk metrik (Pers.(6)). Penurunan secara lengkap seluruh komponen tensor Ricci dan skalar Ricci dapat dilihat pada Lampiran A. Maka dari itu, didapatkan seluruh komponen tensor Ricci yaitu

$$
\begin{aligned}
& R_{00}=-\frac{\Delta Q^{2}}{r^{6}}, \\
& R_{11}=\frac{Q^{2}}{\Delta r^{2}}, \\
& R_{22}=-\frac{Q^{2}}{r^{2}} \\
& R_{33}=-\frac{Q^{2} \sin ^{2} \theta}{r^{2}},
\end{aligned}
$$

dengan skalar Ricci $R$ bernilai nol.

\section{Tensor Einstein dan Tensor Energi Momentum Maxwell}

Diketahui dari Pers.(1) bahwa tensor Einstein $G_{\mu \nu}=$ $R_{\mu \nu}-\frac{1}{2} g_{\mu \nu} R$, sehingga didapatkan seluruh komponen tensor Einstein yaitu

$$
\begin{aligned}
G_{00} & =-\frac{\Delta Q^{2}}{r^{6}}, \\
G_{11} & =\frac{Q^{2}}{\Delta r^{2}}, \\
G_{22} & =-\frac{Q^{2}}{r^{2}}, \\
G_{33} & =-\frac{Q^{2} \sin ^{2} \theta}{r^{2}} .
\end{aligned}
$$

Setelah mencari seluruh komponen tensor Einstein, selanjutnya akan dicari seluruh komponen tensor energi-momentum secara eksplisit. Penurunan secara lengkap tensor energimomentum Maxwell dapat dilihat pada Lampiran B. Maka dari itu, seluruh komponen tensor energi-momentum Maxwell dalam ruangwaktu Pers.(6) adalah

$$
\begin{aligned}
& T_{00}=-\left(\frac{1}{4 \pi}\right) \frac{\Delta Q^{2}}{2 r^{6}}, \\
& T_{11}=\left(\frac{1}{4 \pi}\right) \frac{Q^{2}}{2 \Delta r^{2}}, \\
& T_{22}=-\left(\frac{1}{4 \pi}\right) \frac{Q^{2}}{2 r^{2}}, \\
& T_{33}=-\left(\frac{1}{4 \pi}\right) \frac{Q^{2} \sin ^{2} \theta}{2 r^{2}} .
\end{aligned}
$$

Jika kita mensubstitusikan persamaan Pers.(22)-(25) dan Pers.(26)-(29) ke dalam persamaan medan Einstein-Maxwell (Pers.(4)), dapat dilihat bahwa seluruh komponen tensor Einstein dan tensor energi-momentum Maxwell ini memenuhi Pers.(4).

\section{SIMPULAN}

Dapat disimpulkan bahwa solusi Reissner-Nordström dengan ruangwaktu (6) memenuhi persamaan Einstein-Maxwell (4). Informasi bahwa ada komponen tensor Ricci yang tidak nol yaitu persamaan (18)-(21) menunjukan bahwa metrik (6) adalah bukan solusi vakum persamaan gravitasi Einstein. Ada metode lain untuk mendapatkan solusi Reissner-Nordström, seperti menggunakan bentuk metrik yang lebih umum yaitu misalnya metrik yang statik dan isotropik atau menggunakan prinsip pendekatan variasi seperti pada referensi [2].
[1] A. Einstein, Ann. Phys. (Leipzig), 49, 769-822 (1916).

[2] M.P. Hobson, G.P. Efstathiou, dan A. N. Lasenby, General Relativity: An Introduction for Physicists, (1st ed., Cambridge Univerity Press, New York, 2006).

[3] K. Schwarzschild, Über das Gravitationsfeld eines Massenpunktes nach der Einsteinschen Theorie, Abh. Konigl. Preuss. Akad. Wissenschaften Jahre 1906, 92, Berlin, 1907, 189 (1916).

[4] H. Reissner, Ann. Phys. (Leipzig), 50, 106-120 (1916).

[5] G. Nordström, On the Energy of the Gravitational Field in Einstein's Theory, Proc. Kon. Ned. Akad. Wet. 20, 1238 (1918).

[6] R.P. Kerr, Phys. Rev. Lett., 11, 237-238 (1963).
[7] E.T. Newman and A.I. Janis, J. Math. Phys., 6, 915-917 (1965).

[8] E.T. Newman, et al., J. Math. Phys. 6, 918-919 (1965).

[9] R. Ruffini and J. A. Wheeler, Phys. Today, 24(1), 30-41 (1971).

[10] R.M. Wald, Construction of Metric and Vector Potential Perturbations of a Reissner-Nordstrom Black Hole, Proc. R. Soc. Lond. A369, 67-81 (1979).

[11] P. Pani, E. Berti and L. Gualtieri, Phys. Rev. D, 88, 064048 (2013).

[12] T.P. Cheng, Relativity, Gravitation and Cosmology: A basic introduction (1st ed., Oxford University Press Inc., New York, 2005). 


\section{LAMPIRAN A: TENSOR RICCI UNTUK METRIK REISSNER-NORDSTRÖM}

Komponen tensor Ricci untuk metrik (Pers.(6)) adalah

$$
\begin{aligned}
& R_{00}=-\partial_{\alpha} \Gamma_{00}^{\alpha}+\partial_{0} \Gamma_{0 \alpha}^{\alpha}-\Gamma_{\sigma \alpha}^{\alpha} \Gamma_{00}^{\sigma}+\Gamma_{\sigma 0}^{\alpha} \Gamma_{\alpha 0}^{\sigma} \\
& =-\partial_{1} \Gamma_{00}^{1}-\Gamma_{00}^{1}\left(\Gamma_{10}^{0}+\Gamma_{11}^{1}+\Gamma_{12}^{2}+\Gamma_{13}^{3}\right)+\Gamma_{10}^{0} \Gamma_{00}^{1}+\Gamma_{00}^{1} \Gamma_{10}^{0} \\
& =-\frac{(-2 M+2 r)\left(M r-Q^{2}\right)}{r^{5}}-\frac{\Delta M}{r^{5}}+\frac{5 \Delta M}{r^{6}}-\frac{2\left(M r-Q^{2}\right) \Delta}{r^{6}}+\frac{2\left(M r-Q^{2}\right)^{2}}{r^{6}} \\
& =-\frac{\Delta Q^{2}}{r^{6}} \\
& R_{11}=-\partial_{\alpha} \Gamma_{11}^{\alpha}+\partial_{1} \Gamma_{1 \alpha}^{\alpha}-\Gamma_{\sigma \alpha}^{\alpha} \Gamma_{11}^{\sigma}+\Gamma_{\sigma 1}^{\alpha} \Gamma_{\alpha 1}^{\sigma} \\
& =-\partial_{1} \Gamma_{11}^{1}+\partial_{1} \Gamma_{10}^{0}+\partial_{1} \Gamma_{11}^{1}+\partial_{1} \Gamma_{12}^{2}+\partial_{1} \Gamma_{13}^{3}-\Gamma_{11}^{1}\left(\Gamma_{10}^{0}+\Gamma_{11}^{1}+\Gamma_{12}^{2}+\Gamma_{13}^{3}\right) \\
& +\Gamma_{01}^{0} \Gamma_{01}^{0}+\Gamma_{11}^{1} \Gamma_{11}^{1}+\Gamma_{21}^{2} \Gamma_{21}^{2}+\Gamma_{31}^{3} \Gamma_{31}^{3} \\
& =\frac{M}{r \Delta}-\frac{(-2 M+2 r)\left(M r-Q^{2}\right)}{r \Delta^{2}}-\frac{\left(M r-Q^{2}\right)}{r^{2} \Delta}+\frac{2\left(M r-Q^{2}\right)}{r^{2} \Delta}+\frac{2\left(M r-Q^{2}\right)^{2}}{r^{2} \Delta^{2}} \\
& -\frac{2}{r^{2}}+\frac{2}{r^{2}} \\
& =\frac{Q^{2}}{\Delta r^{2}} \text {. } \\
& R_{22}=-\partial_{\alpha} \Gamma_{22}^{\alpha}+\partial_{2} \Gamma_{2 \alpha}^{\alpha}-\Gamma_{\sigma \alpha}^{\alpha} \Gamma_{22}^{\sigma}+\Gamma_{\sigma 2}^{\alpha} \Gamma_{\alpha 2}^{\sigma} \\
& =-\partial_{1} \Gamma_{22}^{1}+\partial_{2} \Gamma_{23}^{3}-\Gamma_{22}^{1}\left(\Gamma_{10}^{0}+\Gamma_{11}^{1}+\Gamma_{12}^{2}+\Gamma_{13}^{3}\right)+\Gamma_{22}^{1} \Gamma_{12}^{2}+\Gamma_{12}^{2} \Gamma_{22}^{1}+\Gamma_{32}^{3} \Gamma_{32}^{3} \\
& =1-\frac{Q^{2}}{r^{2}}-\csc ^{2} \theta+\frac{2 \Delta}{r^{2}}-\frac{2 \Delta}{r^{2}}+\cot ^{2} \theta \\
& =-\frac{Q^{2}}{r^{2}} \text {. } \\
& R_{33}=-\partial_{\alpha} \Gamma_{33}^{\alpha}+\partial_{3} \Gamma_{3 \alpha}^{\alpha}-\Gamma_{\sigma \alpha}^{\alpha} \Gamma_{33}^{\sigma}+\Gamma_{\sigma 3}^{\alpha} \Gamma_{\alpha 3}^{\sigma} \\
& =-\partial_{1} \Gamma_{33}^{1}-\partial_{2} \Gamma_{33}^{2}-\Gamma_{33}^{1}\left(\Gamma_{10}^{0}+\Gamma_{11}^{1}+\Gamma_{12}^{2}+\Gamma_{13}^{3}\right)-\Gamma_{23}^{3} \Gamma_{33}^{2}+\Gamma_{33}^{\alpha} \Gamma_{\alpha 3}^{3}+\Gamma_{13}^{\alpha} \Gamma_{\alpha 3}^{1}+\Gamma_{23}^{\alpha} \Gamma_{\alpha 3}^{2} \\
& =\frac{(-2 M+2 r) \sin ^{2} \theta}{r}-\frac{\Delta \sin ^{2} \theta}{r^{2}}-\sin ^{2} \theta+\cos ^{2} \theta-\cot \theta \sin \theta \cos \theta \\
& =-\frac{Q^{2} \sin ^{2} \theta}{r^{2}} .
\end{aligned}
$$

dengan skalar Ricci $R$ adalah

$$
\begin{aligned}
R & =g^{\mu \nu} R_{\mu \nu} \\
& =g^{00} R_{00}+g^{11} R_{11}+g^{22} R_{22}+g^{33} R_{33} \\
& =\left(\frac{r^{2}}{\Delta}\right)\left(\frac{-\Delta Q^{2}}{r^{6}}\right)+\left(\frac{-\Delta}{r^{2}}\right)\left(\frac{Q^{2}}{\Delta r^{2}}\right)+\left(\frac{-1}{r^{2}}\right)\left(\frac{-Q^{2}}{r^{2}}\right)+\left(\frac{-1}{r^{2} \sin ^{2} \theta}\right)\left(\frac{-Q^{2} \sin ^{2} \theta}{r^{2}}\right) \\
& =-\frac{Q^{2}}{r^{4}}-\frac{Q^{2}}{r^{4}}+\frac{Q^{2}}{r^{4}}+\frac{Q^{2}}{r^{4}} \\
& =0 .
\end{aligned}
$$




\section{LAMPIRAN B: TENSOR ENERGI-MOMENTUM UNTUK METRIK REISSNER-NORDSTRÖM}

Seluruh komponen tensor energi-momentum Maxwell untuk metrik (Pers.(6)) adalah

$$
\begin{aligned}
& T_{00}=\frac{1}{4 \pi}\left(g^{\gamma \lambda} F_{0 \gamma} F_{0 \lambda}-\frac{1}{4} g_{00} F_{\alpha \beta} F^{\alpha \beta}\right) \\
& =\frac{1}{4 \pi}\left(g^{11} F_{01} F_{01}-\frac{1}{4} g_{00} F_{\alpha \beta} F^{\alpha \beta}\right) \\
& =\frac{1}{4 \pi}\left(\frac{-\Delta}{r^{2}}\left(\frac{-Q}{r^{2}}\right)^{2}-\frac{1}{4}\left(\frac{\Delta}{r^{2}}\right)\left(\frac{-2 Q^{2}}{r^{4}}\right)\right) \\
& =\frac{1}{4 \pi}\left(\frac{-\Delta Q^{2}}{2 r^{6}}\right) \text {. } \\
& T_{11}=\frac{1}{4 \pi}\left(g^{\gamma \lambda} F_{1 \gamma} F_{1 \lambda}-\frac{1}{4} g_{11} F_{\alpha \beta} F^{\alpha \beta}\right) \\
& =\frac{1}{4 \pi}\left(g^{00} F_{10} F_{10}-\frac{1}{4} g_{11} F_{\alpha \beta} F^{\alpha \beta}\right) \\
& =\frac{1}{4 \pi}\left(\frac{r^{2}}{\Delta}\left(\frac{Q}{r^{2}}\right)^{2}-\frac{1}{4}\left(\frac{-r^{2}}{\Delta}\right)\left(\frac{-2 Q^{2}}{r^{4}}\right)\right) \\
& =\frac{1}{4 \pi}\left(\frac{Q^{2}}{2 \Delta r^{2}}\right) \text {. } \\
& T_{22}=\frac{1}{4 \pi}\left(g^{\gamma \lambda} F_{2 \gamma} F_{2 \lambda}-\frac{1}{4} g_{22} F_{\alpha \beta} F^{\alpha \beta}\right) \\
& =\frac{1}{4 \pi}\left(\frac{-1}{4} g_{22} F_{\alpha \beta} F^{\alpha \beta}\right) \\
& =\frac{1}{4 \pi}\left(\frac{-1}{4}\left(-r^{2}\right)\left(\frac{-2 Q^{2}}{r^{4}}\right)\right) \\
& =\frac{1}{4 \pi}\left(\frac{-Q^{2}}{2 r^{2}}\right) \text {. } \\
& T_{33}=\frac{1}{4 \pi}\left(g^{\gamma \lambda} F_{3 \gamma} F_{3 \lambda}-\frac{1}{4} g_{33} F_{\alpha \beta} F^{\alpha \beta}\right) \\
& =\frac{1}{4 \pi}\left(\frac{-1}{4} g_{33} F_{\alpha \beta} F^{\alpha \beta}\right) \\
& =\frac{1}{4 \pi}\left(\frac{-1}{4}\left(-r^{2} \sin ^{2} \theta\right)\left(\frac{-2 Q^{2}}{r^{4}}\right)\right) \\
& =\frac{1}{4 \pi}\left(\frac{-Q^{2} \sin ^{2} \theta}{2 r^{2}}\right) \text {. }
\end{aligned}
$$

